\title{
Anti-Unwinding Attitude Control with Fixed-Time Convergence for a Flexible Spacecraft
}

\author{
Chutiphon Pukdeboon ${ }^{1}$ and Anuchit Jitpattanakul ${ }^{2}$ \\ ${ }^{1}$ Nonlinear Dynamic Analysis Research Center, Department of Mathematics, Faculty of Applied Science, \\ King Mongkut's University of Technology North Bangkok, Bangkok 10800, Thailand \\ ${ }^{2}$ Department of Mathematics, Faculty of Applied Science, King Mongkut's University of Technology North Bangkok, \\ Bangkok 10800, Thailand \\ Correspondence should be addressed to Chutiphon Pukdeboon; cpd@kmutnb.ac.th
}

Received 12 April 2017; Accepted 21 June 2017; Published 7 August 2017

Academic Editor: Vaios Lappas

Copyright @ 2017 Chutiphon Pukdeboon and Anuchit Jitpattanakul. This is an open access article distributed under the Creative Commons Attribution License, which permits unrestricted use, distribution, and reproduction in any medium, provided the original work is properly cited.

\begin{abstract}
This paper investigates the fixed-time attitude tracking control problem for flexible spacecraft with unknown bounded disturbances. First, with the knowledge of norm upper bounds of external disturbances and the coupling effect of flexible modes, a novel robust fixed-time controller is designed to deal with this problem. Second, the controller is further enhanced by an adaptive law to avoid the knowledge of norm upper bounds of external disturbances and coupling effect of flexible modes. This control law guarantees the convergence of attitude tracking errors in fixed time where the settling time is bounded by a constant independent of initial conditions. Moreover, the proposed controllers can prevent the unwinding phenomenon. Simulation results are presented to demonstrate the performance of the proposed control scheme.
\end{abstract}

\section{Introduction}

With the development of modern satellite technology, space missions are expected to ensure rapid, high-precision and global response such as navigation, communication, astronomy, and earth observations. Modern spacecraft often requires the structure of a rigid hub with flexible appendages. Owing to strong coupling between hub and flexible appendages attitude maneuver is affected by vibration of flexible appendages. This may degrade pointing accuracy and dynamic performance of the attitude control system. Furthermore, in practical control systems, there inevitably exist model uncertainty and nonlinearity, inertia uncertainty, and various external disturbance torques, such as aerodynamic torque, radiation torque, and gravity gradient torque. These factors make the design of an attitude control law with rapid and precise maneuver performance for a flexible spacecraft very difficult and even pose a great challenge to space missions. In recent years, a variety of control methods have been proposed to solve the attitude control problem, like proportional derivative control [1], adaptive control [2], $H_{\infty}$ control [3], passivity-based control $[4,5]$, sliding mode control [6-8], active disturbance rejection control [9], disturbance observer based control [10], and so forth [11-13]. Although these nonlinear control laws have offered sufficient and reliable effectiveness and robustness in spacecraft attitude control systems, they require infinite time to accomplish an attitude maneuver. However, the ability of rapid maneuver is highly required in many real-time space missions. In other words, infinite-time attitude control is inadequate in some space missions.

In fact, finite-time stabilization [14-17] can offer faster convergence to the origin and better disturbance rejection than asymptotic stabilization. Thus, finite-time control approaches are desirable to be considered in spacecraft attitude controller designs. Terminal sliding mode control (TSMC) $[18,19]$ method has provided a practical way to design a finite-time controller. For TSMC, nonlinear sliding 
surface was proposed and the finite-time convergence was analyzed based on the concept of terminal attractor. TSMC has been applied to spacecraft attitude control problems [2022]. Later, a nonsingular terminal sliding mode based robust finite-time control law was proposed in $[23,24]$ to deal with the singularity problem, which exists in traditional TSMC. More recently, nonsingular fast terminal sliding mode control laws for a rigid spacecraft were designed in $[25,26]$. In $[27,28]$ nonsingular fast terminal sliding mode control laws have been developed for attitude motions of flexible spacecraft.

Apart from the above attention to achieve finite-time convergence, actuator fault is another key issue in spacecraft attitude control which may cause the performance degradation or even result in control system instability. To handle this issue, fault-tolerant control (FTC) is a widely used scheme to enhance the capability of maintaining control stability and high performance despite casual actuator failures. Recently, several FTC schemes have been developed for spacecraft attitude control [29-32]. Xiao et al. [33] proposed a fault-tolerant controller for a flexible spacecraft in the presence of possible additive fault and partial loss of actuator effectiveness fault. Zhang et al. [34] presented a robust fault-tolerant control scheme to achieve attitude control of flexible spacecraft with disturbances and actuator failures. These control algorithms can tolerate partial loss of actuator effectiveness. In [35] a fault-tolerant sliding mode attitude controller for flexible spacecraft with inertia uncertainty, external disturbance, actuator misalignment, and input saturation has been developed. Recently, FTC design for spacecraft attitude control with consideration of different kinds of actuator faults and failures is still an open problem.

Although control methods mentioned above can provide good results of attitude tracking control of spacecraft, the initial system state must be known to estimate the settling time. It would be very useful if the settling time can be determined without knowledge of initial conditions. In [36, 37] Polyakov and his colleagues have proposed the concept of the fixed-finite-time stability. For the fixed-finite-time stability concept, the upper bound of the settling time can be estimated and it is independent of initial conditions. For the spacecraft attitude control system it is desirable to estimate the settling time independent of initial conditions. Moreover, these control methods may cause the unwinding phenomenon encountered in unit quaternion based attitude systems since they consider only one of two equilibrium points of unit quaternion [38].

In this paper, the fixed-time control problem associated with attitude tracking of flexible spacecraft in the presence of bounded external disturbances and coupling effect of flexible modes is investigated. The aim is to design a robust fixed-time attitude controller so that rapid attitude maneuver can be achieved with high pointing accuracy. By extending the concepts of fixed-finite stability by Polyakov and his colleagues $[36,37]$, a new result of fixed-finite stability is introduced. This result is also used to develop our proposed controllers. So far, to the best our knowledge, there were no results of fixedtime sliding control methods for attitude maneuver control of a flexible spacecraft in presence of bounded disturbances with an unknown boundary. Inspired by the above discussion, two fixed-time controllers are presented in this paper and the main contributions lie in the following aspects:

(1) A new result of fixed-time stability is proposed by extending concepts of fixed-finite stability by Polyakov and his colleagues [36, 37]. For the case that the upper bounds of disturbances and the coupling effect of flexible modes are available in advance, a novel fixed-time controller is proposed for flexible spacecraft attitude maneuver. This controller ensures the convergence of attitude tracking errors in fixed time where the settling time is bounded by a constant independent of initial conditions. Moreover, this controller can eliminate the unwinding phenomenon.

(2) A new adaptive law is designed to estimate the upper bounds of the disturbances and the coupling effect in fixed time. Then, a new adaptive fixed-time controller without requiring prior knowledge of their boundaries is designed. By Lyapunov stability theory, rigorous fixed-time stability is analyzed and the expression of an accurate upper bound of convergent regions is provided.

The remaining part of this paper is organized as follows. Section 2 presents the description of a flexible spacecraft model and some key lemmas. Then, a robust fixed-time controller is designed in Section 3. The fixed-time stability proof is also given. In Section 4, a novel robust adaptive fixedtime attitude controller for flexible spacecraft is designed. The developed attitude controller makes the system states converge into a small neighborhood of the designed sliding mode in fixed time. To verify the effectiveness of the proposed control methods, simulation results are given in Section 5. In Section 6, we present conclusions.

\section{Nonlinear Model of Spacecraft and Problem Formulation}

2.1. Mathematical Model of a Flexible Spacecraft. The flexible spacecraft is characterized by a central rigid body with attached appendages. The mathematical model of the flexible spacecraft can be described in the body-fixed frame as [39]

$$
\begin{aligned}
J \dot{\omega}+\omega^{\times}\left(J \omega+\delta^{T} \dot{\eta}\right)+\delta^{T} \ddot{\eta} & =u(t)+\tau_{d}(t), \\
\ddot{\eta}+C \dot{\eta}+K \eta & =-\delta \dot{\omega}, \\
\dot{Q} & =\frac{1}{2}\left[\begin{array}{c}
-q^{T} \\
q_{0} I_{3}+q^{\times}
\end{array}\right] \omega,
\end{aligned}
$$

where $J \in R^{3 \times 3}$ is the symmetric inertia matrix of the whole structure, $\omega \in R^{3}$ is the angular velocity of the spacecraft in the body frame, $\tau_{d} \in R^{3}$ denotes external disturbances, $u \in$ $R^{3}$ is the control torque acting on the hub, $\eta \in R^{n}$ is the modal coordinate vector of the flexible appendages, $n$ is the number of flexible modes considered, and $\delta \in R^{3 \times n}$ is the coupling 
matrix between the flexible appendage and the hub. In (2), the damping matrix $C$ and stiffness matrix $K$ are given by

$$
\begin{aligned}
& C=\left[\begin{array}{ccc}
2 \zeta_{1} \Lambda_{1} & \cdots & 0 \\
\vdots & \ddots & \vdots \\
0 & \cdots & 2 \zeta_{n} \Lambda_{n}
\end{array}\right], \\
& K=\left[\begin{array}{ccc}
\Lambda_{1}^{2} & \cdots & 0 \\
\vdots & \ddots & \vdots \\
0 & \cdots & \Lambda_{n}^{2}
\end{array}\right],
\end{aligned}
$$

where $\zeta_{i}$ and $\Lambda_{i}$ denote the damping ration and natural frequency of the $i$ th order mode. The operator $(\cdot)^{\times}$denotes a $3 \times 3$ symmetric matrix such as

$$
\omega^{\times}=\left[\begin{array}{ccc}
0 & -\omega_{3} & \omega_{2} \\
\omega_{3} & 0 & -\omega_{1} \\
-\omega_{2} & \omega_{1} & 0
\end{array}\right],
$$

for $\omega=\left[\begin{array}{lll}\omega_{1} & \omega_{2} & \omega_{3}\end{array}\right]^{T}$. The vector $Q=\left[\begin{array}{ll}q_{0} & q^{T}\end{array}\right]^{T}$ represents the attitude quaternion of the spacecraft subject to the unity length constraint, that is, $\|Q\|=1$, where $q_{0}$ and $q=$ $\left[\begin{array}{lll}q_{1} & q_{2} & q_{3}\end{array}\right]$ denote the scalar and the vector components of the unit quaternion, respectively. $I_{3}$ is the $3 \times 3$ identity matrix. In (2), we define an auxiliary variable $\vartheta=\delta \omega+\dot{\eta}$ and one has

$$
\dot{\vartheta}=\delta \dot{\omega}+\ddot{\eta}=-C \vartheta+C \delta \omega-K \eta .
$$

Substituting (6) into (1) yields

$$
\left(J-\delta^{T} \delta\right) \dot{\omega}=-\omega^{\times} J \omega+u(t)+\xi(t)+\tau_{d}(t),
$$

where the total coupling effect term $\xi(t)$ denotes

$$
\xi(t)=\delta^{T}\left[\begin{array}{ll}
K & C
\end{array}\right]\left[\begin{array}{l}
\eta \\
\vartheta
\end{array}\right]-\delta^{T} C \delta \omega-\omega^{\times} \delta^{T}(\vartheta-\delta \omega) .
$$

Let $Q_{d}=\left[\begin{array}{ll}q_{d 0} & q_{d}^{T}\end{array}\right]^{T}$ with $q_{d}=\left[\begin{array}{lll}q_{d 1} & q_{d 2} & q_{d 3}\end{array}\right]^{T}$ be the unit quaternion representing the desired attitude and satisfying $\left\|Q_{d}\right\|=1$. Let $\omega_{r} \in R^{3}$ be the desired angular velocity. The quaternion error $Q_{e}=\left[\begin{array}{ll}e_{0} & e^{T}\end{array}\right]^{T}$ with $e=\left[\begin{array}{lll}e_{1} & e_{2} & e_{3}\end{array}\right]^{T}$ and the angular velocity error $\omega_{e}$ are defined as follows:

$$
\begin{aligned}
e & =q_{d 0} q-q_{d}^{\times} q-q_{0} q_{d}, \\
e_{0} & =q^{T} q_{d}+q_{0} q_{d 0}, \\
\omega_{e} & =\omega-\omega_{r} .
\end{aligned}
$$

The unit quaternion $Q_{e}$ satisfies $\left\|Q_{e}\right\|=1$.

In fact, due to onboard payload motion, rotation of solar arrays, fuel consumption, and out-gassing during operation, the inertial matrix $J$ of spacecraft may be time-varying. Here, we assume that it consists of two parts; that is, $J=J_{n}+\Delta J$, where $J_{n}$ and $\Delta J$ represent the nominal value component and the parameter perturbation component of the inertial matrix $J$, respectively. Both of the nominal value component of $J_{n}$ and the perturbation matrix $\Delta J$ are symmetric since $J$ is always a symmetric matrix.

If the terms $\Delta J \dot{\omega}-\omega^{\times} \Delta J \omega$ are considered as the disturbance, then (1) becomes

$$
\left(J_{n}-\delta^{T} \delta\right) \dot{\omega}_{e}=-J_{n} \dot{\omega}_{r}-\omega^{\times} J_{n} \omega+u(t)+\xi(t)+d(t),
$$

where $d(t)=-\Delta J \dot{\omega}-\omega^{\times} \Delta J \omega+\tau_{d}(t)$.

Under the coordinate given in (9), (1) and (3) can be written as

$$
\begin{aligned}
J_{0} \dot{\omega}_{e} & =-J_{0} \dot{\omega}_{r}-\omega^{\times} J_{n} \omega+u(t)+\xi(t)+d(t), \\
\dot{Q}_{e} & =\frac{1}{2}\left[\begin{array}{c}
-e^{T} \\
e_{0} I_{3}+e^{\times}
\end{array}\right] \omega_{e}
\end{aligned}
$$

where $J_{0}=J_{n}-\delta^{T} \delta$.

Throughout the paper, it is assumed that the influence caused by the external disturbances and the flexible modes is bounded in the following sense.

Assumption 1. The total external disturbance $d(t)$ in (11) is bounded and satisfies $\|d\| \leq \theta_{0}$, where $\theta_{0}>0$ is an unknown constant.

Assumption 2. The coupling effect term $\xi(t)$ in (11) satisfies $\|\xi(t)\| \leq b_{1}+b_{2}\|\omega\|^{2}$ with $b_{1}>0$ and $b_{2}>0$. In other words, $\|\xi(t)\| \leq \theta_{1} \Phi_{1}$ for $\Phi_{1}=1+\|\omega\|^{2}$ and $\theta_{1}=\max \left(b_{1}, b_{2}\right)$.

2.2. Definition and Lemma. Consider the following autonomous system:

$$
\begin{aligned}
\dot{x}(t) & =f(x(t)), \\
x(0) & =0, \\
f(0) & =0, \\
x & \in R^{n},
\end{aligned}
$$

where $f: U_{0} \rightarrow R^{n}$ is continuous on an open neighborhood $U_{0}$ of the origin $x=0$.

Definition 3 (see [16]). The equilibrium $x=0$ of system (13) is finite-time convergent if there exist an open neighborhood $U \subset U_{0}$ of the origin and a function and the function $T$ : $U \backslash\{0\} \rightarrow(0, \infty)$ such that the solution of the system (13) defined as $x\left(t, x_{0}\right)$ with $x_{0} \in U \backslash\{0\} \subset R^{n}$ satisfies $x\left(t, x_{0}\right) \in$ $U \backslash\{0\} \subset R^{n}$ for $t \in\left[0, T\left(x_{0}\right)\right]$ and $\lim _{t \rightarrow T\left(x_{0}\right)} x\left(t, x_{0}\right)=0$ with $x\left(t, x_{0}\right)=0$ for $t>T\left(x_{0}\right)$. The zero solution of the system (13) is finite-time stable if it is Lyapunov stable and finite-time convergent in a $U \subset U_{0}$ of the origin. If $U_{0}=U=R^{n}$, then the zero solution is globally finite-time stable.

Lemma 4 (see [16]). For system (13), suppose that there exists a continuous positive definite function $V(x): U \rightarrow R$ such that

$$
\dot{V}(x)+\beta V(x)^{p} \leq 0, \quad x \in U_{0} \backslash\{0\},
$$

where $p \in(0,1), \beta \in R^{+}$, and $U_{0} \subset U$ is an open neighborhood of the origin. Then, the origin is a finite-time 
stable equilibrium of system (13). Furthermore, $T\left(x_{0}\right)$ is the settling time that is defined as the time needed to reach $V(x) \equiv$ 0 and satisfies

$$
T\left(x_{0}\right) \leq \frac{1}{\beta(1-p)} V\left(x_{0}\right)^{1-p},
$$

where $V\left(x_{0}\right)$ is the initial value of $V(x)$.

Definition 5 (see [36]). The equilibrium $x=0$ of system (13) is said to be fixed-finite-time stable if it is globally finite-time stable and the settling-time function $T\left(x_{0}\right)$ is bounded, that is, a positive constant $T_{\max }$ can be found in such a way that $T\left(x_{0}\right) \leq T_{\text {max }}, \forall x_{0} \in R^{n}$.

Lemma 6 (see [22]). For system (13), suppose there exists a positive definite and radially unbounded function $V(x): R^{n} \rightarrow$ $R$ such that $V(x)=0 \Rightarrow x=0$ and

$$
\dot{V}(x) \leq-\alpha V^{p}(x)-\beta V(x)^{q},
$$

where $\alpha>0, \beta>0, p \in(0,1)$, and $q>1$. Then, the origin of system (13) is fixed-time stable and

$$
T\left(x_{0}\right) \leq \frac{1}{\alpha(1-p)}+\frac{1}{\beta(q-1)}, \quad \forall x_{0} \in R^{n} .
$$

Lemma 7 (see [40]). Let $x_{i} \in R, i=1,2, \ldots, n$ be real numbers and $p \in(0,1)$; then the following inequality holds:

$$
\left(\left|x_{1}\right|+\cdots+\left|x_{n}\right|\right)^{p} \leq\left|x_{1}\right|^{p}+\cdots+\left|x_{n}\right|^{p} .
$$

Lemma 8 (see [41]). For any numbers $x_{i} \geq 0, i=1,2, \ldots, n$, and $p>1$, then

$$
\sum_{i=1}^{n} x_{i}^{p} \geq n^{1-p}\left(\sum_{i=1}^{n} x_{i}\right)^{p}
$$

In the following proposition, we give a new result of fixedfinite stability. This result is an extended version of fixed-finite stability presented in Lemma 6 and will be used in controller designs in later section.

Proposition 9. Consider system (13). If there is a positive definite and radially unbounded function $V(x): R^{n} \rightarrow R$ such that $V(x)=0 \Rightarrow x=0$ and

$$
\dot{V}(x) \leq-\gamma V(x)-\alpha V^{p}(x)-\beta V(x)^{q},
$$

where $\alpha>0, \beta>0, p \in(0,1)$, and $q>1$, then, the origin of system (13) is fixed-time stable

$$
\begin{aligned}
T\left(x_{0}\right) \leq \frac{1}{\beta(q-1)}+\frac{1}{\beta(1-p)} \ln \left(\frac{\gamma+\alpha}{\alpha}\right) & \\
& \\
& \forall x_{0} \in R^{n} .
\end{aligned}
$$

Proof. Owing to (20), we can obtain

$$
\dot{V}(x(t)) \leq \begin{cases}-\gamma V(x)-\beta V^{p}(x), & V(x(t)) \leq 1, \\ -\beta V^{q}(x), & V(x(t))>1 .\end{cases}
$$

Hence, for any $x(t)$ such that $V(x(0))>1$, the last inequality ensures $V\left(x\left(t_{0}\right)\right) \leq 1$. This means $V(x(0))$ is reduced such that $V(x(t))=1$ at the time $t_{0}$. Integrating both sides of the last inequality yields

$$
V^{1-q}(t) \leq-(1-q) \beta t+V^{1-q}(x(0)),
$$

and consequently we can obtain $t_{0}$ as

$$
t_{0} \leq \frac{1-V^{1-q}(x(0))}{\beta(q-1)} .
$$

As is known that $V(x(0))>1$, one has

$$
t_{0} \leq \frac{1}{\beta(q-1)} .
$$

Next, the first inequality ensures that $V\left(x\left(t_{0}\right)\right)=1$ will approach $V\left(x\left(t_{1}\right)\right)=0$. Now, $t_{1}$ can be calculated from the first inequality. The first inequality can be rearranged as

$$
V^{-p}(x(t)) \dot{V}(x(t)) \leq-\gamma V^{1-p}(x(t))-\alpha,
$$

and consequently

$$
d t \leq-\frac{V^{-p}(x(t))}{\gamma V^{1-p}(x(t))+\alpha} d V(x(t)) .
$$

Now, integrating both sides of the above inequality from $t_{0}$ to $t_{1}$ yields

$$
\begin{aligned}
t_{1}-t_{0} & \leq-\int_{V\left(x\left(t_{0}\right)\right)}^{0} \frac{V^{-p}(x(t))}{\gamma V^{1-p}(x(t))+\alpha} d V(x(t)) \\
& =-\frac{1}{\gamma(1-p)}\left[\ln \alpha-\ln \left(\gamma V^{1-p}\left(x\left(t_{0}\right)+\alpha\right)\right)\right] \\
& =\frac{1}{\gamma(1-p)} \ln \left(\frac{\gamma V^{1-p}\left(x\left(t_{0}\right)\right)+\alpha}{\alpha}\right) .
\end{aligned}
$$

Using $V\left(x\left(t_{0}\right)\right)=1$, one obtains

$$
\begin{aligned}
t_{1} & \leq t_{0}+\frac{1}{\gamma(1-p)} \ln \left(\frac{\gamma+\alpha}{\alpha}\right) \\
\text { or } t_{1} & \leq \frac{1}{\beta(q-1)}+\frac{1}{\gamma(1-p)} \ln \left(\frac{\gamma+\alpha}{\alpha}\right) .
\end{aligned}
$$

This completes the proposition.

2.3. Problem Statement. In this paper, $q_{d}, \omega_{d}$, and $\dot{\omega}_{d}$ are assumed to be bounded, and the quaternion and angular velocity measurements are always available. The main objective is to design a control law which forces the attitude and angular velocity errors to a small region around the origin in finite time in the sense of a fixed-time concept. This can be expressed as

$$
\lim _{t \rightarrow T}\left(e(t), \omega_{e}(t)\right)=0
$$

where $T$ is the convergence time that can be estimated even if information of initial values of system states is unavailable. 


\section{Fixed-Time Attitude Controller}

In this section, a new fixed-time sliding surface is introduced. The fixed-time convergence of this sliding surface is analyzed. Then, a new fixed-time based sliding mode attitude controller is developed to achieve rapid maneuver and high-precise attitude control performance for a flexible spacecraft in presence of external disturbances.

Now, we define a new variable $z\left(\omega_{e}, e_{0}, e\right)$ as

$$
z\left(\omega_{e}, q_{e 0}, q_{e}\right)=\omega_{e}+\operatorname{sign}\left(e_{0}\right) K e,
$$

where $K=\operatorname{diag}\left(k_{1}, k_{2}, k_{3}\right)$ is a positive diagonal matrix.

Inspired by [22], we developed a nonsingular terminal sliding surface for the spacecraft attitude system described by (11) and (12) as follows:

$$
s=z+C_{1} \int_{0}^{t} \operatorname{sign}(z)^{\beta} d \tau+C_{2} \int_{0}^{t} \operatorname{sign}(z)^{\gamma} d \tau,
$$

where $C_{1}=\operatorname{diag}\left(c_{11}, c_{12}, c_{13}\right)$ and $C_{2}=\operatorname{diag}\left(c_{21}, c_{22}, c_{23}\right)$ are constant matrices with $c_{1 i}, c_{2 i}>0(i=1,2,3), 0<\beta<1$, and $\gamma>1$. For any vector $x=\left[\begin{array}{lll}x_{1} & x_{2} & x_{3}\end{array}\right]^{T}$ and constant $p>0$, the function of $\operatorname{sign}(x)^{p}$ is defined as

$$
\operatorname{sign}(x)^{p}=\left[\begin{array}{l}
\left|x_{1}\right|^{p} \operatorname{sign}\left(x_{1}\right) \\
\left|x_{2}\right|^{p} \operatorname{sign}\left(x_{2}\right) \\
\left|x_{3}\right|^{p} \operatorname{sign}\left(x_{3}\right)
\end{array}\right] .
$$

For the sake simplicity, the sliding surface can be rewritten in the scalar form $(i=1,2,3)$ as

$$
\begin{aligned}
s_{i}= & z_{i}+c_{1 i} \int_{0}^{t}\left|z_{i}\right|^{\beta} \operatorname{sign}\left(z_{i}\right) d \tau \\
& +c_{2 i} \int_{0}^{t}\left|z_{i}\right|^{\gamma} \operatorname{sign}\left(z_{i}\right) d \tau .
\end{aligned}
$$

When the sliding mode is established under a suitably designed controller, one can obtain

$$
\begin{aligned}
s_{i}= & z_{i}+c_{1 i} \int_{0}^{t}\left|z_{i}\right|^{\beta} \operatorname{sign}\left(z_{i}\right) d \tau \\
& +c_{2 i} \int_{0}^{t}\left|z_{i}\right|^{\gamma} \operatorname{sign}\left(z_{i}\right) d \tau=0, \\
\dot{s}_{i}= & \dot{z}_{i}+c_{1 i}\left|z_{i}\right|^{\beta} \operatorname{sign}\left(z_{i}\right)+c_{2 i}\left|z_{i}\right|^{\gamma} \operatorname{sign}\left(z_{i}\right)=0 .
\end{aligned}
$$

Therefore, the dynamics of the associate sliding mode can be obtained as

$$
\begin{aligned}
\dot{z}_{i}=-c_{1 i}\left|z_{i}\right|^{\beta} \operatorname{sign}\left(z_{i}\right)-c_{2 i}\left|z_{i}\right|^{\gamma} \operatorname{sign}\left(z_{i}\right), & \\
& (i=1,2,3) .
\end{aligned}
$$

Theorem 10. The zero solution $z_{i}=0(i=1,2,3)$, of the sliding mode dynamics (36), is globally fixed-time stable and the settling time is given by

$$
T \leq \frac{1}{c_{1 i}(1-\beta)}+\frac{1}{c_{2 i}(\gamma-1)} .
$$

Proof. The Lyapunov function candidate is chosen as

$$
V_{1}=\left|z_{i}\right|
$$

Its first time derivative is

$$
\begin{aligned}
\dot{V}_{1} & =\operatorname{sign}\left(z_{i}\right) \dot{z}_{i} \\
& =\operatorname{sign}\left(z_{i}\right)\left(-c_{1 i}\left|z_{i}\right|^{\beta} \operatorname{sign}\left(z_{i}\right)-c_{2 i}\left|z_{i}\right|^{\gamma} \operatorname{sign}\left(z_{i}\right)\right) \\
& =-c_{1 i}\left|z_{i}\right|^{\beta}-c_{2 i}\left|z_{i}\right|^{\gamma}=-c_{1 i} V_{1}^{\beta}-c_{2 i} V_{1}^{\gamma} .
\end{aligned}
$$

By Lemma 6, it can be concluded that $z_{i}=0(i=1,2,3)$ are fixed-time stabilized. This completes the proof.

Once the fixed-time-based sliding surface is designed, it is followed by designing a control law $u \in R^{3}$ as follows:

$$
\begin{aligned}
u= & \omega^{\times} J_{n} \omega+J_{0} \dot{\omega}_{r}+J_{0} K \operatorname{sign}\left(e_{0}\right) \frac{1}{2}\left(e_{0} I_{3}+e^{\times}\right) \omega_{e} \\
& -J_{0} C_{1} \operatorname{sign}(z)^{\beta}-J_{0} C_{2} \operatorname{sign}(z)^{\gamma}-\mu_{1} s \\
& -\mu_{2} \operatorname{sign}(s)-\mu_{3} \operatorname{sign}(s)^{\rho},
\end{aligned}
$$

where $\rho>1, \mu_{1}=\operatorname{diag}\left(\mu_{11}, \mu_{12}, \mu_{13}\right), \mu_{2}=\operatorname{diag}\left(\mu_{21}, \mu_{22}, \mu_{23}\right)$, and $\mu_{3}=\operatorname{diag}\left(\mu_{31}, \mu_{32}, \mu_{33}\right)$ are diagonal matrices with $\mu_{1 i}, \mu_{2 i}, \mu_{3 i}>0(i=1,2,3)$. For any vector $x=\left[\begin{array}{lll}x_{1} & x_{2} & x_{3}\end{array}\right]^{T}$, the function of $\operatorname{sign}(x)$ is defined as

$$
\operatorname{sign}(x)=\left[\begin{array}{l}
\operatorname{sign}\left(x_{1}\right) \\
\operatorname{sign}\left(x_{2}\right) \\
\operatorname{sign}\left(x_{3}\right)
\end{array}\right],
$$

where $\gamma_{1} \in(0,1)$ and $k_{1}$ is a positive number; the larger the value of $k_{1}$, the faster the reaching speed. The term $\mu_{2} \operatorname{sign}(s)$ is employed to cope with external disturbances and coupling effect.

The fixed-time stability of the closed-loop system under the action of the controller (40) is analyzed in the following theorem.

Theorem 11. Consider the system described by (11) and (12) and let Assumptions 1 and 2 hold. If the control law is designed as (40), then the sliding surfaces $=0$ is achieved in a fixed time.

Proof. According to (32), we can obtain the time derivative of $J_{0} s$ along the trajectory of the system consisting of (11) and (12):

$$
\begin{aligned}
J_{0} \dot{s}= & J_{0} \dot{\omega}_{e}+J_{0} K \operatorname{sign}\left(e_{0}\right)+J_{0} C_{1} \operatorname{sign}(z)^{\beta} \\
& +J_{0} C_{2} \operatorname{sign}(z)^{\gamma} .
\end{aligned}
$$

Then, substituting (11) into (42) yields

$$
\begin{aligned}
J_{0} \dot{s}= & J_{0} \dot{z}+J_{0} C_{1} \operatorname{sign}(z)^{\beta}+J_{0} C_{2} \operatorname{sign}(z)^{\gamma} \\
= & -\omega^{\times} J_{n} \omega-J_{0} \dot{\omega}_{r}+u+d+\xi \\
& +J_{0} K \operatorname{sign}\left(e_{0}\right) \frac{1}{2}\left(e_{0} I_{3}+e^{\times}\right) \omega_{e} \\
& +J_{0} C_{1} \operatorname{sign}(z)^{\beta}+J_{0} C_{2} \operatorname{sign}(z)^{\gamma}
\end{aligned}
$$


Substituting (40) into (43), we obtain

$$
J_{0} \dot{s}=-\mu_{1} s-\mu_{2} \operatorname{sign}(s)-\mu_{3} \operatorname{sign}(s)^{\rho}+d+\xi .
$$

Consider the candidate Lyapunov function:

$$
V_{2}=\frac{1}{2} s^{T} J_{0} s
$$

which satisfies

$$
\lambda_{\min }\left(J_{0}\right)\|s\|^{2} \leq 2 V_{2} \leq \lambda_{\max }\left(J_{0}\right)\|s\|^{2},
$$

where $\lambda_{\min }\left(J_{0}\right)$ and $\lambda_{\max }\left(J_{0}\right)$ denote the minimum and maximum singular values of the matrix $J_{0}$.

According to (44), the derivative of $V_{2}$ along the trajectory of the system described by (11) and (12) is

$$
\begin{aligned}
\dot{V}_{2}= & s^{T} J_{0} \dot{s} \\
= & s^{T}\left(-\mu_{1} s-\mu_{2} \operatorname{sign}(s)-\mu_{3} \operatorname{sign}(s)^{\rho}+d\right) \\
= & -\lambda_{\min }\left(\mu_{1}\right) \sum_{i=1}^{3}\left(\left|s_{i}\right|\right)-\lambda_{\min }\left(\mu_{2}\right) \sum_{i=1}^{3}\left|s_{i}\right|^{2} \\
& -\lambda_{\min }\left(\mu_{3}\right) \sum_{i=1}^{3}\left|s_{i}\right|^{1+\rho}+\|s\|\|d\| \\
\leq & -\left(\lambda_{\min }\left(\mu_{1}\right)-\theta_{0}\right)\|s\|-\lambda_{\min }\left(\mu_{2}\right)\|s\|^{2} \\
& -3^{(1-\rho) / 2} \lambda_{\min }\left(\mu_{3}\right)\|s\|^{1+\rho} .
\end{aligned}
$$

Letting $\varepsilon=\lambda_{\min }\left(\mu_{1}\right)-\theta_{0}>0$, one obtains

$$
\begin{aligned}
\dot{V}_{2} \leq & -\frac{\sqrt{2} \varepsilon}{\sqrt{\lambda_{\max }(J)}} V_{2}^{1 / 2}-\frac{2 \lambda_{\min }\left(\mu_{2}\right)}{\lambda_{\max }(J)} V_{2} \\
& -3^{(1-\rho) / 2} \lambda_{\min }\left(\mu_{3}\right)\left(\frac{2}{\lambda_{\max }(J)}\right)^{(1+\rho) / 2} V_{2}^{(1+\rho) / 2} \\
= & -\kappa_{1} V_{2}^{1 / 2}-\kappa_{2} V_{2}-\kappa_{3} V_{2}^{(1+\rho) / 2},
\end{aligned}
$$

where $\kappa_{1}=\sqrt{2} \varepsilon / \sqrt{\lambda_{\max }\left(J_{0}\right)}, \kappa_{2}=2 \lambda_{\min }\left(\mu_{2}\right) / \lambda_{\max }\left(J_{0}\right)$, and $\kappa_{3}=\lambda_{\min }\left(\mu_{3}\right)\left(3^{(1-\rho) / 2}\right)\left(2 / \lambda_{\max }\left(J_{0}\right)\right)^{(1+\rho) / 2}$. Hence, the sliding variable $s(t)$ reaches zero in a fixed time that can be calculated by using Proposition 9.

Remark 12. To avoid chattering in practical applications, owing to the imperfect implementation of the sign function, a saturation function is a simple choice to replace the sign function in the control law (40). Thus, in (40), for $s=\left[\begin{array}{lll}s_{1} & s_{2} & s_{3}\end{array}\right]^{T}$, $\operatorname{sign}(s)$ is replaced by $\operatorname{sat}(s)=\left[\begin{array}{lll}\operatorname{sat}\left(s_{1}\right) & \operatorname{sat}\left(s_{2}\right) & \operatorname{sat}\left(s_{3}\right)\end{array}\right]^{T}$. The function $\operatorname{sat}(\cdot)$ is a saturation function

$$
\operatorname{sat}(x)= \begin{cases}1, & x>\phi_{s} \\ x, & |x|<\phi_{s} \\ -1, & x<-\phi_{s}\end{cases}
$$

where $\phi_{s}$ is a small positive constant.

\section{Adaptive Fixed-Time Attitude Control}

In the previous section, the proposed fixed-time attitude controller (40) can force the states of the system described by (11) and (12) to the origin. However, it is required to know the upper bounds of external disturbances and coupling effect. In practice, it is difficult to know these upper bounds, which implies that the values of $\theta_{0}$ and $\theta_{1}$ may not be available. Without prior knowledge of the upper bounds of external disturbances and coupling effect, the gain of sign function needs to be chosen sufficiently large to satisfy the existence condition of sliding mode, which may reduce the steady-state performance of the attitude control system due to undesirable chattering in control torque and require large control input magnitudes.

In order to achieve more efficiently performance, an adaptive scheme is constructed to estimate the upper bound information. Then, an adaptive law is designed to guarantee fixed-time reachability of given desired attitude motion. Here, we design an adaptive fixed-time-based control law as

$$
\begin{aligned}
u= & \omega^{\times} J_{n} \omega+J_{0} \dot{\omega}_{r}+J_{0} K \operatorname{sign}\left(e_{0}\right) \frac{1}{2}\left(e_{0} I_{3}+e^{\times}\right) \omega_{e} \\
& -J_{0} C_{1} \operatorname{sign}(z)^{\beta}-J_{0} C_{2} \operatorname{sign}(z)^{\gamma}-\mu_{1} s \\
& -\mu_{2} \operatorname{sign}(s)-\mu_{2} \operatorname{sign}(s)^{\rho}+u_{a},
\end{aligned}
$$

where $u_{a}$ is a continuous adaptive control term designed as follows:

$$
u_{a}=-\left(\frac{\widehat{\theta}_{0}}{2 \varepsilon_{0}^{2}}+\frac{\widehat{\theta}_{1} \Phi}{2 \varepsilon_{1}^{2}}\right) s
$$

where

$$
\begin{aligned}
& \dot{\hat{\theta}}_{0}=\beta_{0}\left(\frac{\|s\|^{2}}{2 \varepsilon_{0}^{2}}-k_{0} \widehat{\theta}_{0}\right), \\
& \dot{\hat{\theta}}_{1}=\beta_{1}\left(\frac{\Phi\|s\|^{2}}{2 \varepsilon_{1}^{2}}-k_{1} \widehat{\theta}_{1}\right) .
\end{aligned}
$$

Theorem 13. Consider the motion equations (11) and (12) that satisfy Assumptions 1 and 2 , but with $\bar{d}$ and $\theta_{1}$ unknown. If the control law is designed as (50), then the attitude error e and the angular velocity error $\omega_{e}$ can be stabilized in fixed time into a neighborhood of the sliding mode $s(t)=0$.

Proof. Consider the following candidate Lyapunov function:

$$
V_{3}=\frac{1}{2}\left(s^{T} J_{0} s+\frac{1}{\beta_{0}} \widetilde{\theta}_{0}^{2}+\frac{1}{\beta_{1}} \widetilde{\theta}_{1}^{2}\right),
$$


where $\widetilde{\theta}_{0}$ and $\widetilde{\theta}_{1}$ are the parameter estimation errors defined as $\widetilde{\theta}_{0}:=\theta_{0}-\widehat{\theta}_{0}$ and $\widetilde{\theta}_{1}:=\theta_{1}-\widehat{\theta}_{1}$, respectively. The derivative of $V_{3}$ along the trajectory of system (11) and (12) is

$$
\begin{aligned}
\dot{V}_{3} & =s^{T} J_{0} \dot{s}+\frac{1}{\beta_{0}} \widetilde{\theta}_{0} \dot{\hat{\theta}}_{0}+\frac{1}{\beta_{1}} \widetilde{\theta}_{1} \dot{\hat{\theta}}_{1}=s^{T}\left(-\mu_{1} s\right. \\
& -\mu_{2} \operatorname{sign}(s)-\mu_{3} \sin (z)^{\rho}-\frac{\widehat{\theta}_{0}}{2 \varepsilon_{0}^{2}} s-\frac{\widehat{\theta}_{1} \Phi}{2 \varepsilon_{1}^{2}} s+d(t) \\
& +\xi(t))-\left(\frac{\|s\|^{2}}{2 \varepsilon_{0}^{2}}-k_{0} \widehat{\theta}_{0}\right)\left(\theta_{0}-\widehat{\theta}_{0}\right)-\left(\frac{\Phi\|s\|^{2}}{2 \varepsilon_{1}^{2}}\right. \\
& \left.-k_{1} \widehat{\theta}_{1}\right)\left(\theta_{1}-\widehat{\theta}_{1}\right) \leq-s^{T} \mu_{1} s-s^{T} \mu_{2} \operatorname{sign}(s)-s^{T} \mu_{3} \\
& +\operatorname{sign}(s)^{\rho}+\|s\|\|d\|+\|s\|\|\xi(t)\|-\frac{\theta_{0}}{2 \varepsilon_{0}^{2}}\|s\|^{2} \\
& -\frac{\theta_{1}}{2 \varepsilon_{1}^{2}}\|s\|^{2} \Phi+k_{0} \widehat{\theta}_{0} \widetilde{\theta}_{0}+k_{1} \widehat{\theta}_{1} \widetilde{\theta}_{1} \leq-\lambda_{\min }\left(\mu_{1}\right)\|s\|^{2} \\
& +\|s\|\|d\|+\theta_{1}\|s\| \Phi-\frac{\theta_{0}}{2 \varepsilon_{0}^{2}}\|s\|^{2}-\frac{\theta_{1}}{2 \varepsilon_{1}^{2}}\|s\|^{2} \Phi \\
& +k_{0} \widehat{\theta}_{0} \widetilde{\theta}_{0}+k_{1} \widehat{\theta}_{1} \widetilde{\theta}_{1} .
\end{aligned}
$$

Note that

$$
\begin{aligned}
k_{0} \widehat{\theta}_{0} \widetilde{\theta}_{0} & =k_{0} \theta_{0} \widetilde{\theta}_{0}-k_{0} \widetilde{\theta}_{0}^{2} \leq k_{0}\left(-\widetilde{\theta}_{0}^{2}+\frac{1}{2} \widetilde{\theta}_{0}^{2}+\frac{1}{2} \theta_{0}^{2}\right) \\
& =-\frac{k_{0}}{2} \widetilde{\theta}_{0}^{2}+\frac{k_{0}}{2} \theta_{0}^{2},
\end{aligned}
$$

and similarly

$$
k_{1} \widehat{\theta}_{1} \widetilde{\theta}_{1} \leq-\frac{k_{1}}{2} \widetilde{\theta}_{1}^{2}+\frac{k_{1}}{2} \theta_{1}^{2} .
$$

Moreover, letting $\theta_{0}=(\bar{d})^{2}$ and applying Young's inequality, one obtains

$$
\begin{gathered}
\|s\|\|d\| \leq \frac{\theta_{0}}{2 \varepsilon_{0}^{2}}\|s\|^{2}+\frac{\varepsilon_{0}^{2}}{2}, \\
\theta_{1}\|s\| \Phi \leq \frac{\theta_{1}}{2 \varepsilon_{1}^{2}}\|s\|^{2} \Phi+\frac{\varepsilon_{1}^{2}}{2} .
\end{gathered}
$$

Differentiating $V_{4}$ with respect to time, one obtains

$$
\begin{aligned}
\dot{V}_{4} \leq & -s^{T} \mu_{1} s-s^{T} \mu_{2} \operatorname{sign}(s)-s^{T} \mu_{3} \operatorname{sign}(s)^{\rho} \\
& -\frac{\widehat{\theta}_{0}}{2 \varepsilon_{0}^{2}}\|s\|^{2}-\frac{\hat{\theta}_{1} \Phi}{2 \varepsilon_{1}^{2}}\|s\|^{2}+\|s\|\|d\|+\|s\|\|\xi\| \\
\leq & -\lambda_{\min }\left(\mu_{1}\right)\|s\|^{2}-\lambda_{\min }\left(\mu_{2}\right)\|s\| \\
& -\lambda_{\min }\left(\mu_{3}\right) \sum_{i=1}^{3}\left|s_{i}\right|^{\rho}-\frac{\widehat{\theta}_{0}}{2 \varepsilon_{0}^{2}}\|s\|^{2}-\frac{\widehat{\theta}_{1}}{2 \varepsilon_{1}^{2} \Phi}\|s\|^{2} \\
& +\frac{\theta_{0}}{2 \varepsilon_{0}^{2}}\|s\|^{2}+\frac{\theta_{1}}{2 \varepsilon_{1}^{2} \Phi}\|s\|^{2}+\frac{\varepsilon_{0}^{2}}{2}+\frac{\varepsilon_{1}^{2}}{2} \\
\leq & -\lambda_{\min }\left(\mu_{1}\right)\|s\|^{2}-\lambda_{\min }\left(\mu_{2}\right)\|s\| \\
& -\lambda_{\min }\left(\mu_{3}\right) \sum_{i=1}^{3}\left|s_{i}\right|^{\rho}-\frac{\widetilde{\theta}_{0}}{2 \varepsilon_{0}^{2}}\|s\|^{2}-\frac{\widetilde{\theta}_{1}}{2 \varepsilon_{1}^{2} \Phi}\|s\|^{2} \\
& +\frac{\varepsilon_{0}^{2}}{2}+\frac{\varepsilon_{1}^{2}}{2} \\
\leq & -\left(\lambda_{\min }\left(\mu_{1}\right)-\frac{\bar{\theta}_{0}}{2 \varepsilon_{0}^{2}}-\frac{\bar{\theta}_{1} \Phi}{2 \varepsilon_{1}^{2}}\right)\|s\|^{2} \\
& -\lambda_{\min }\left(\mu_{2}\right)\|s\|-3^{(1-\rho) / 2} \lambda_{\min }\left(\mu_{3}\right)\|s\|^{\rho+1}+\omega
\end{aligned}
$$

here $\alpha=\min \left(2 \lambda_{\min }\left(\mu_{1}\right) / \lambda_{\max }\left(J_{0}\right), k_{0} \beta_{0}, k_{1} \beta_{1}\right)$. ultimate bounded (UUB). Therefore, one can assume that $\widetilde{\theta}_{0} \leq \bar{\theta}_{0}$ and $\widetilde{\theta}_{1} \leq \bar{\theta}_{1}$. To prove the fixed-time stability, we define a new Lyapunov function

$$
V_{4}=\frac{1}{2} s^{T} J_{0} s .
$$

where $\Upsilon=\varepsilon_{0}^{2} / 2+\varepsilon_{1}^{2} / 2+\left(k_{0} / 2\right) \theta_{0}^{2}+\left(k_{1} / 2\right) \theta_{1}^{2}$. As a result, one has 
$3^{(1-\rho) / 2} \lambda_{\min }\left(\mu_{3}\right)>\omega /\|s\|^{\rho+1}$, the trajectories of the solution of the system converge to the residual set given by

$$
\begin{aligned}
D & =\left\{\lim _{t \rightarrow T_{f}} s(t) \mid\|s\|\right. \\
& \left.\leq \min \left(\frac{\omega}{\lambda_{\min }\left(\mu_{2}\right)},\left(\frac{\omega}{3^{(1-\rho) / 2} \lambda_{\min }\left(\mu_{3}\right)}\right)^{1 /(1+\rho)}\right)\right\} .
\end{aligned}
$$

Hence, the proof of Theorem 13 is completed.

Remark 14. In particular, $\hat{\theta}_{0}$ and $\hat{\theta}_{1}$ are the estimated values of $\theta_{0}$ and $\theta_{1}$, in Assumptions 1 and 2, respectively. In [13], the upper bound of $d(t)$ was estimated by traditional adaptive update law. The sign function was combined with the adaptive update law and it can only ensure uniformly ultimate boundedness of $\hat{\theta}_{0}$ and $\hat{\theta}_{1}$. In this paper, the design parameters $k_{0}$ and $k_{1}$ are used to determine the convergence region, and these parameters can be selected to be sufficiently small. However, these parameters also determine the convergence speed of the estimated bounds $\widehat{\theta}_{0}$ and $\hat{\theta}_{1}$. If values $k_{0}$ and $k_{1}$ are chosen too small, then a slow convergence rate is obtained. Therefore, a compromise is made between the convergence region and the convergence speed. Moreover, the values of $k_{0}$ and $k_{1}$ cannot be selected too small [42]. Based on the above adaptive law, the trajectories of $\hat{\theta}_{0}$ and $\widehat{\theta}_{1}$ are reduced significantly and then converge to a value near zero.

\section{Simulations}

Numerical simulations on the flexible spacecraft have been conducted to verify the performance of the proposed adaptive fixed-time control law (50) and PD-based sliding mode control (PD-SMC) method in [5]. For this, the same model parameters used in [39] are applied in this study. The inertia matrix and the flexible coupling matrix are as follows:

$$
J=\left[\begin{array}{ccc}
350 & 3 & 4 \\
3 & 270 & 10 \\
4 & 10 & 190
\end{array}\right] \mathrm{kg} \cdot \mathrm{m}^{2}
$$

and coupling matrices

$$
\delta=\left[\begin{array}{ccc}
6.45637 & 1.27814 & 2.15629 \\
-1.25619 & 0.91756 & -1.67264 \\
1.11678 & 2.48901 & -0.83674 \\
1.23637 & -2.6581 & -1.12503
\end{array}\right] \mathrm{kg}^{1 / 2} \cdot \mathrm{m} / \mathrm{s}^{2},
$$

respectively. The natural frequencies and damping are provided by

$$
\begin{aligned}
& \Lambda_{1}=0.7681 \\
& \Lambda_{2}=1.1038 \\
& \Lambda_{3}=1.8733, \\
& \Lambda_{4}=2.5496 \mathrm{rad} / \mathrm{sec}
\end{aligned}
$$

$$
\begin{aligned}
& \zeta_{1}=0.0056 \\
& \zeta_{2}=0.0086 \\
& \zeta_{3}=0.013 \\
& \zeta_{1}=0.025
\end{aligned}
$$

In this numerical simulation, we assume that the desired angular velocity is given by

$$
\omega_{r}(t)=0.05\left[\begin{array}{c}
\sin \left(\frac{\pi t}{100}\right) \\
\sin \left(\frac{2 \pi t}{100}\right) \\
\sin \left(\frac{3 \pi t}{100}\right)
\end{array}\right] \mathrm{rad} / \mathrm{s} .
$$

For the initial conditions of the unit quaternion and the target unit quaternion, we set $Q(0)=$ $\left[\begin{array}{llll}-0.17365 & -0.2632 & 0.7896 & -0.5264\end{array}\right]^{T}$ and $Q_{d}(0)=$ $\left[\begin{array}{llll}0 & 0 & 0 & 1\end{array}\right]^{T}$, respectively. The initial value of the angular velocity is supposed to be $\omega(0)=\left[\begin{array}{lll}0 & 0 & 0\end{array}\right]^{T} \mathrm{rad} / \mathrm{s}$. We bounded the magnitude of the control torque as $\left|u_{i}\right| \leq 3.0 \mathrm{~N}-\mathrm{m}, i=1,2,3$. The external disturbance torque that includes constant disturbance and periodic disturbance of two different frequencies is described as follows:

$$
\begin{aligned}
\tau_{d}(t)= & {\left[\begin{array}{c}
3 \cos (t)-10+4 \sin (0.3 t) \\
3 \cos (0.5 t)+15-1.5 \sin (0.2 t) \\
3 \sin (t)+10+8 \sin (0.4 t)
\end{array}\right] } \\
& \times 10^{-2} \mathrm{Nm} .
\end{aligned}
$$

The parameters for the controller (50) are chosen as $K=$ $0.2 I_{3}, C_{1}=I_{3}, C_{1}=0.6 I_{3}, \beta=7 / 9, \gamma=1.1, \mu_{1}=5 I_{3}, \mu_{2}=$ $3 I_{3}, \mu_{3}=1.2 I_{3}, \rho=5 / 3, \eta_{0}=10, \eta_{1}=10, \varepsilon_{0}=0.01$, and $\varepsilon_{1}=0.01$. For the PD-SMC method in [5], simulations are performed with the parameters given as $k_{P}=3, k_{d}=8$, $k_{e}=3.5$, and $\Gamma=20$.

The performance of the PD-SMC method in [5] is shown in Figures 1-7. From Figure 1 one can see that the time responses of quaternion errors converge to zero after 60 seconds. Figure 2 depicts the unwinding phenomenon encountered in unit quaternion based attitude systems. Since $e_{0}(0)=-0.17365$, the scalar quaternion error should finally converge to the equilibrium point $e_{0}(t)=-1$ instead of the equilibrium point $e_{0}(t)=1$. As shown in Figure 3 , the time responses of angular velocity tracking errors are stabilized to zero but they are not smooth due to coupling effect of flexible modes. Also, Figure 4 shows that the time responses of sliding variables $s_{i}(i=1,2,3)$ converge to zero with low accuracy. As shown in Figure 5, the time responses of control torques have much variation during the first 60 seconds. Figures 6 and 7 illustrate the time responses of modal displacements which are ultimately bounded by small values near zero. For the tracking accuracy, the Euclidean norm of steady errors of $e(t), \omega_{e}(t)$, and $s(t)$ can be listed as $\left\|q_{e}\right\| \leq 5.7 \times 10^{-3}$ and $\left\|\omega_{e}\right\| \leq$ $7.7 \times 10^{-4}$ and $\|s\| \leq 1.77 \times 10^{-4}$ with sampling time $h=0.005$. 


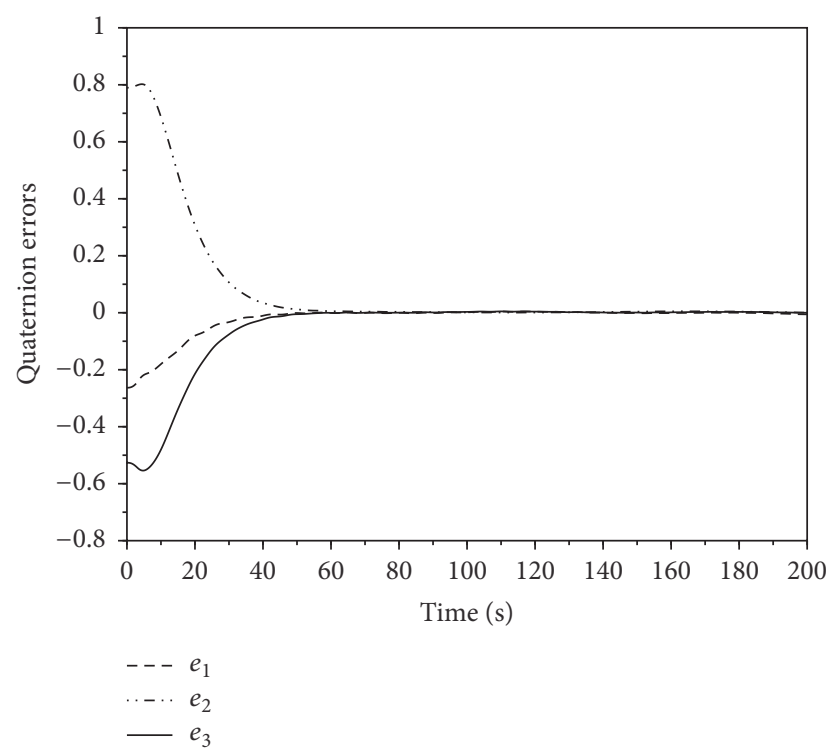

FIgURE 1: Quaternion errors under PD-SMC.

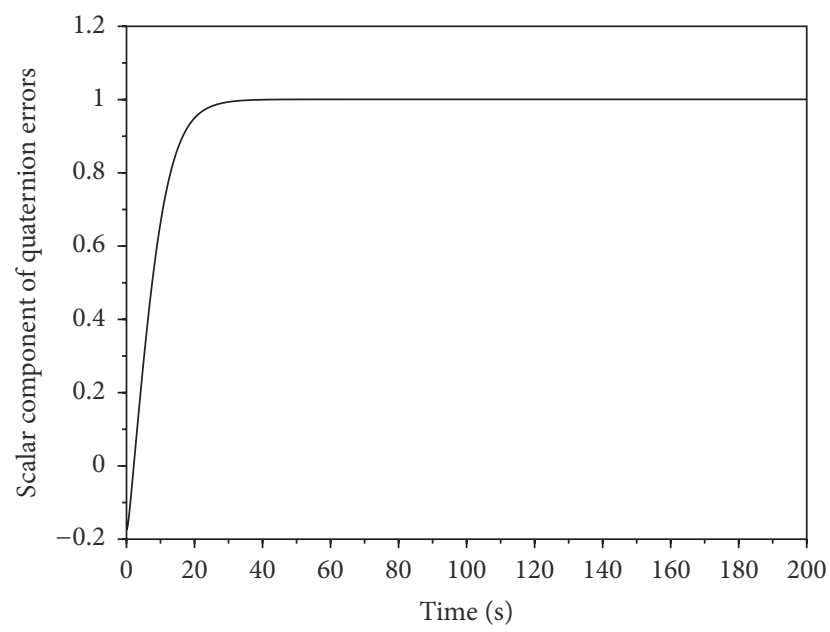

FIgURE 2: Scalar component of quaternion errors under PD-SMC.

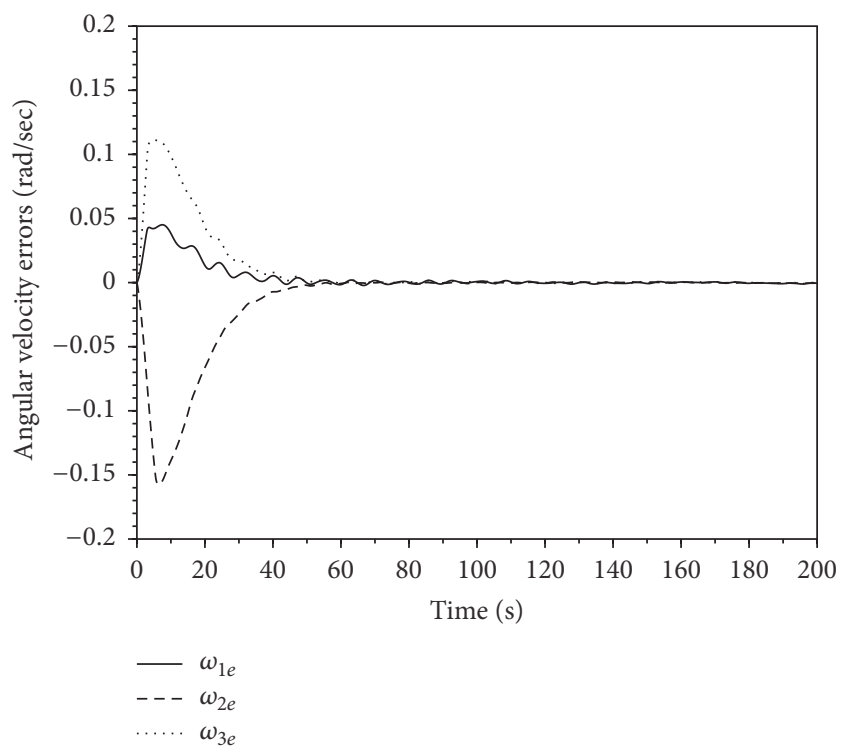

Figure 3: Angular velocity errors under PD-SMC.

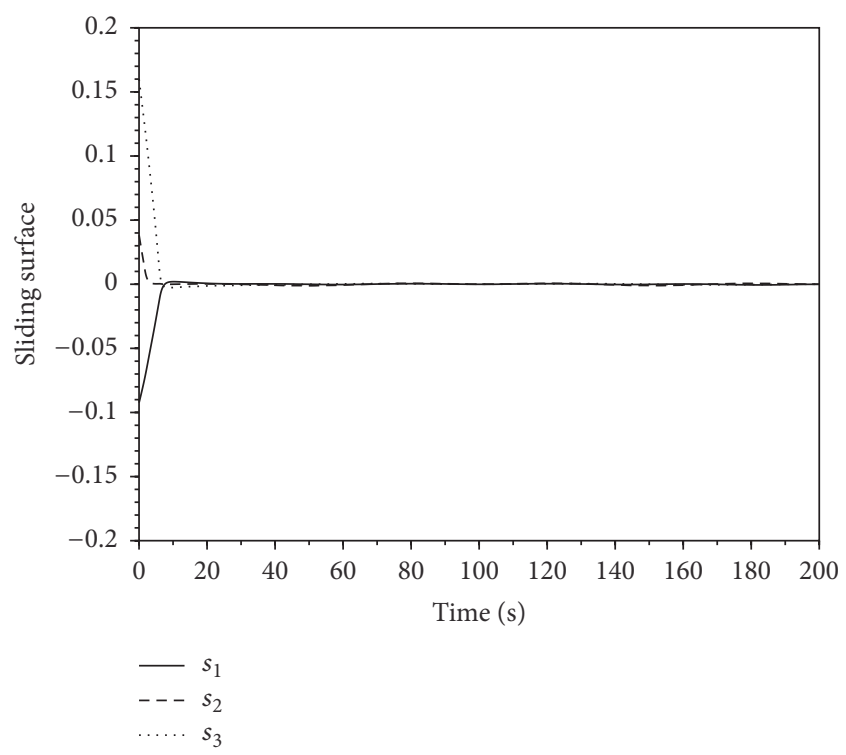

FIGURE 4: Sliding surface under PD-SMC.

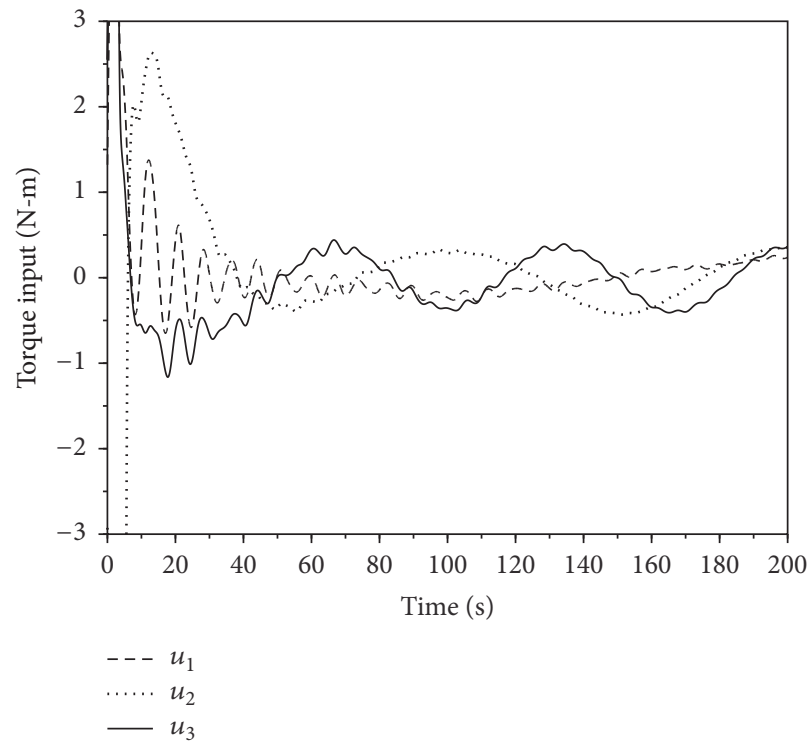

Figure 5: First and second modes under PD-SMC.

Figures 8-14 show the performance of the proposed adaptive fixed-time control law (50). As can be seen from Figure 8, time responses of quaternion errors can be stabilized to zero. Figure 9 shows that the controller (50) can prevent the unwinding phenomenon. When $e_{0}(0)=-0.17365$, the scalar quaternion errors can properly converge to the equilibrium point $e_{0}(t)=-1$. As shown in Figure 10, the time responses of angular velocity errors are smoother when compared with those obtained by the PD-SMC method in [5]. From Figure 11 one can see that the sliding surface $s=0$ is achieved with higher precision than those of the PD-SMC method. Figure 12 shows the time responses of control torques which have small variation. As shown in Figures 13 and 14 the flexible mode vibration is reduced greatly at the beginning 


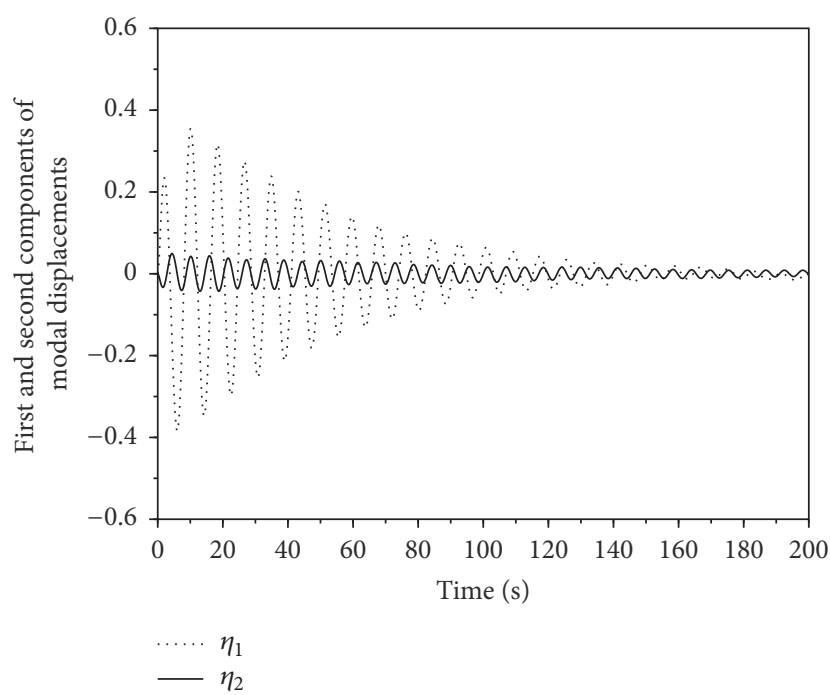

FIGURE 6: First and second modes under PD-SMC.

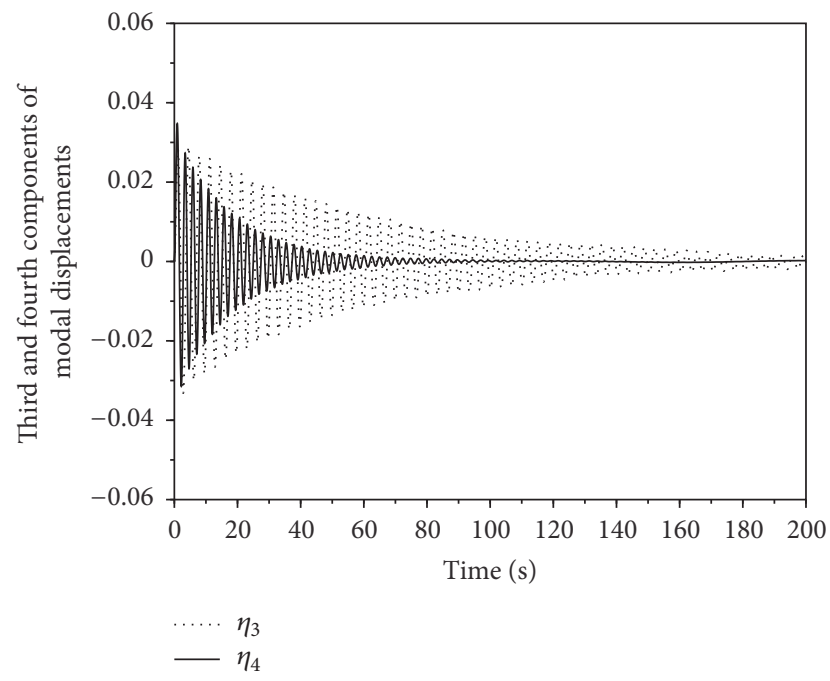

FIgURE 7: Third and fourth modes under PD-SMC.

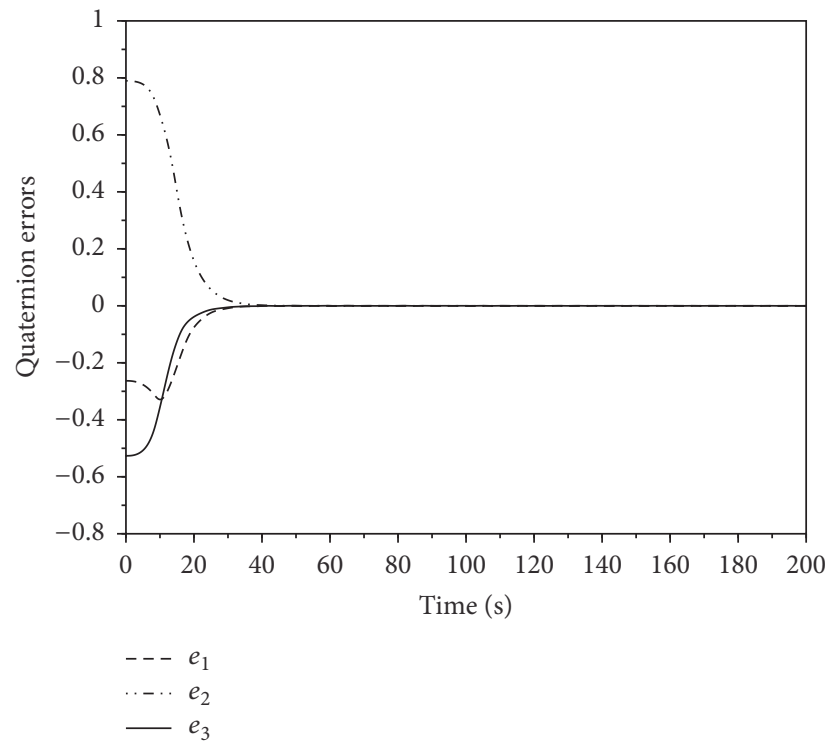

FIGURE 8: Quaternion errors under controller (50).

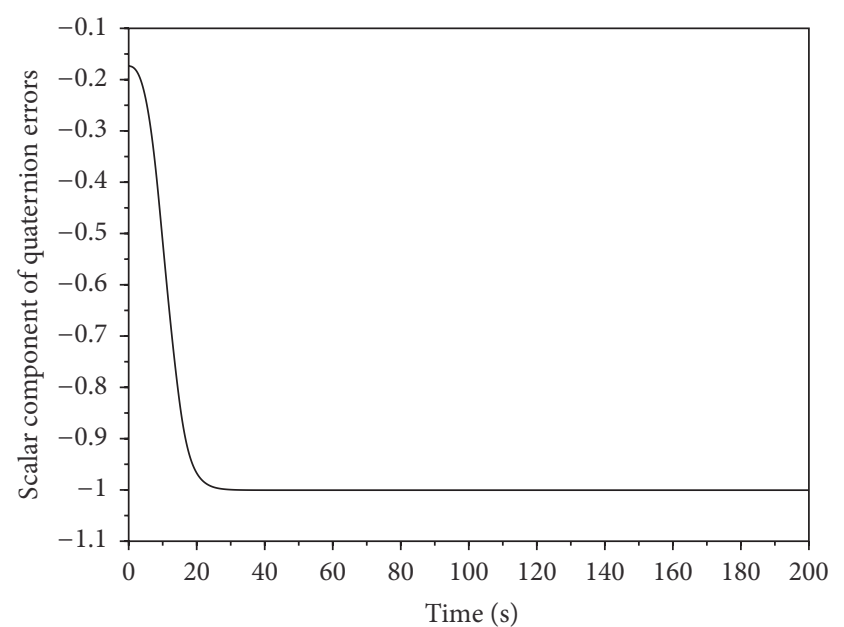

FIGURE 9: Scalar component of quaternion errors under controller (50).

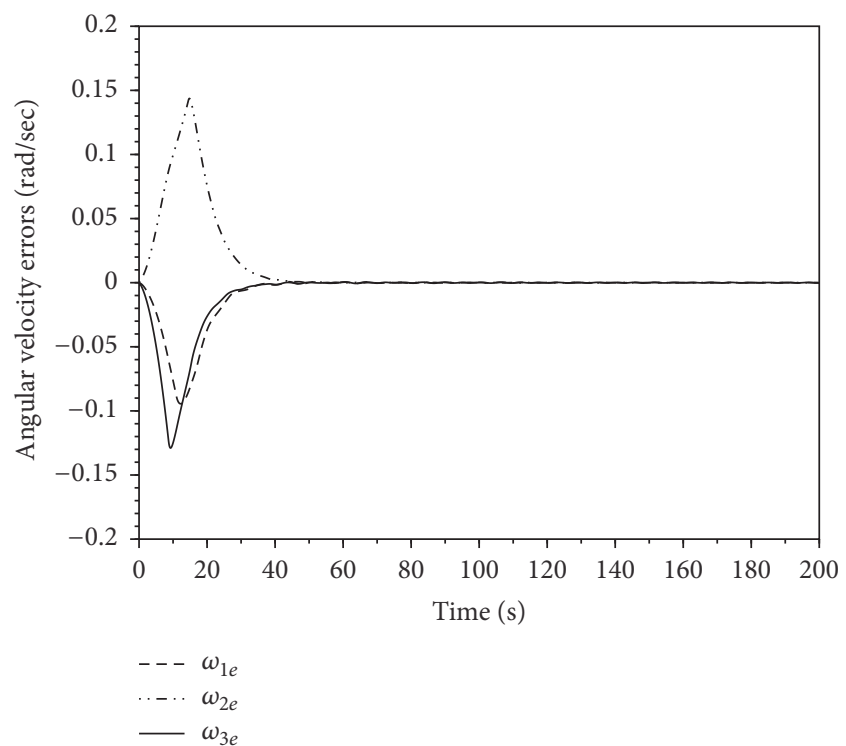

FIGURE 10: Angular velocity errors under controller (50).

and then decreased to a small value. In fact these modal coordinates converge to a small amplitude of the flexible mode vibration (less than 0.05) after 50 seconds. Practically, it is sufficiently good for some spacecraft missions. However, sufficiently large control torques can be applied to keep the flexible mode vibration at an acceptable amplitude of the flexible mode vibration in a small amount of time. Regarding the tracking accuracy, the Euclidean norm of steady errors of $e(t), \omega_{e}(t)$, and $s(t)$ can be listed as $\left\|q_{e}\right\| \leq 4.6 \times 10^{-5}$ and $\left\|\omega_{e}\right\| \leq 2.06 \times 10^{-4}$ and $\|s\| \leq 1.87 \times 10^{-4}$ with sampling time $h=0.005$.

From these simulation results, the proposed adaptive fixed-time control law (50) provides offers smoother time responses of attitude and angular velocity errors and higher accuracy of attitude and angular velocity errors than the PD-SMC method in [5]. Furthermore, the controller (50) 


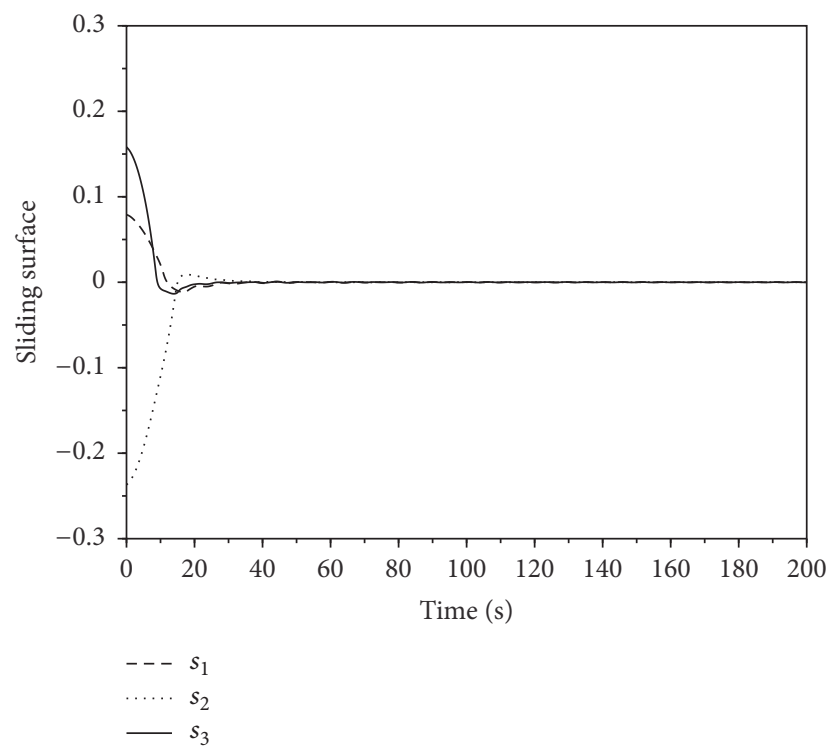

FIGURE 11: Sliding surface under controller (50).

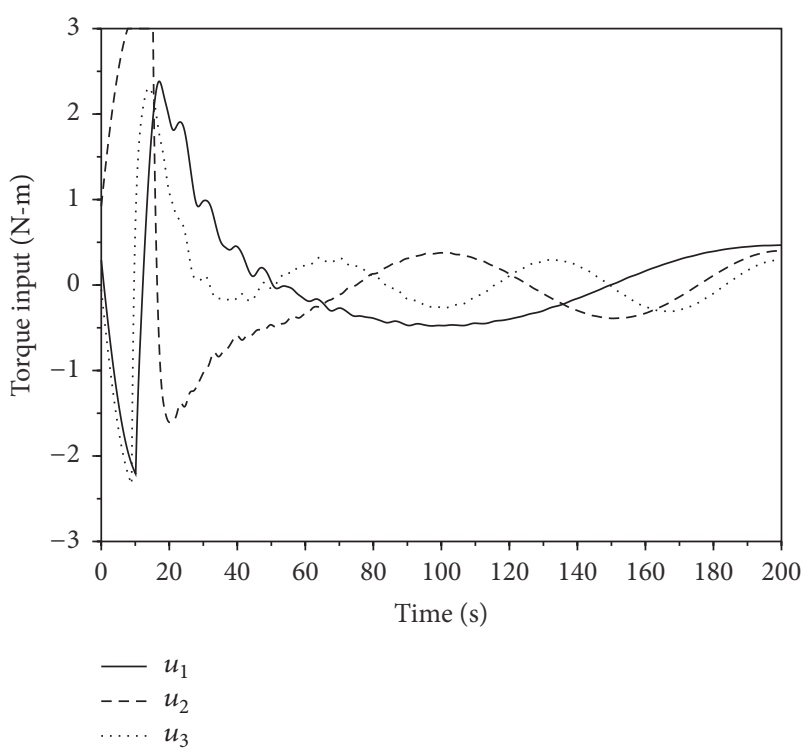

FIGURE 12: Control inputs under controller (50).

effectively prevents the unwinding phenomenon. In view of the simulation, the proposed control law seems to be a more effective control approach for general case of attitude tracking maneuvers of a flexible spacecraft.

\section{Conclusion}

In this paper, an adaptive fixed-time control law has been developed for the attitude tracking control problem of a flexible spacecraft. The first objective of this research has been aimed at providing a new result in fixed-time stability. Then, a fixed-time attitude tracking controller is proposed in the presence of external disturbances and coupling effect of flexible modes. Later, the controller is further enhanced

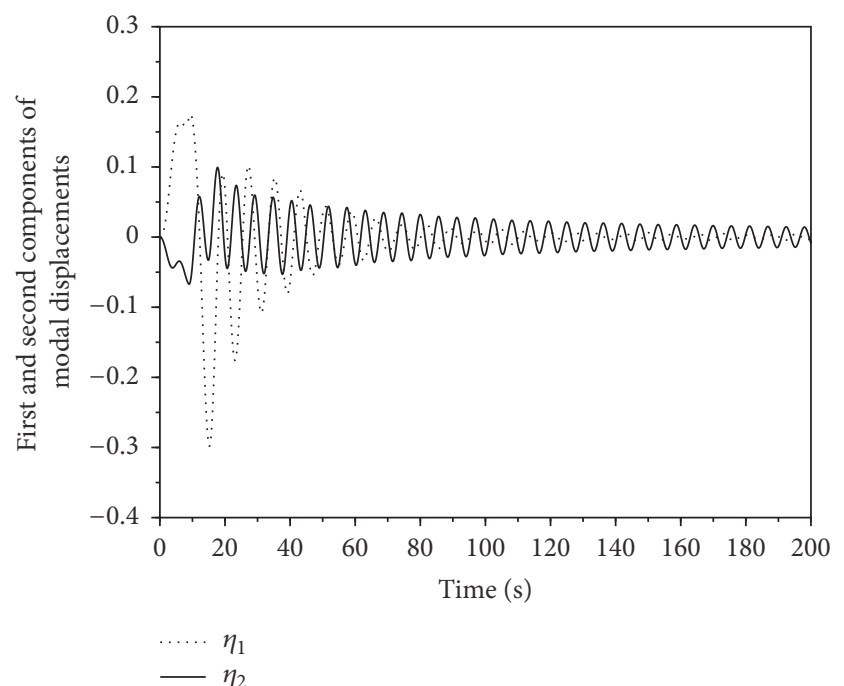

Figure 13: First and second modes under controller (50).

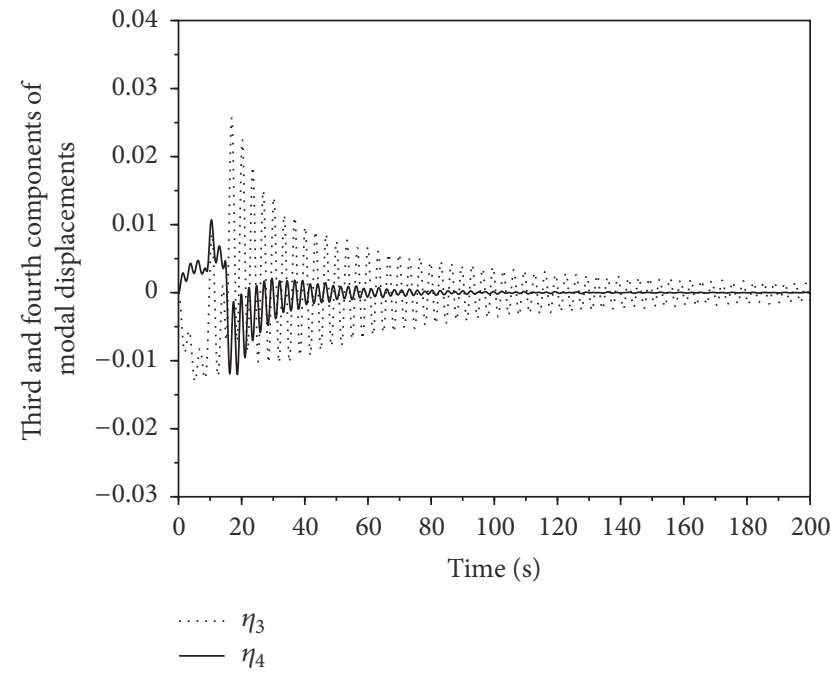

Figure 14: Third and fourth modes under controller (50).

by an adaptive law to relax the knowledge of norm upper bounds of external disturbances and coupling effect of flexible modes. The proposed adaptive fixed-time controller offers a fast transient process and high attitude tracking accuracy. This controller can avoid the unwinding phenomenon. Using Lyapunov stability theory, we have proved that the error dynamics converge to a desired region containing the origin in finite time in the sense of fixed-time convergence. Numerical simulations on attitude control of a flexible spacecraft model are also presented to demonstrate the performance of the proposed control methods.

\section{Conflicts of Interest}

The authors declare that there are no conflicts of interest regarding the publication of this paper. 


\section{Acknowledgments}

This research was funded by King Mongkut's University of Technology North Bangkok, Contract no. KMUTNB-60GOV-61.

\section{References}

[1] H. Liu, L. Guo, and Y. M. Zhang, "An anti-disturbance PD control scheme for attitude control and stabilization of flexible spacecrafts," Nonlinear Dynamics, vol. 67, no. 3, pp. 2081-2088, 2012.

[2] K. Lu and Y. Q. Xia, "Adaptive attitude tracking control for rigid spacecraft with finite-time convergence," Automatica, vol. 49, no. 12, pp. 3591-3599, 2013.

[3] R. Zhang, T. Li, and L. Guo, "Disturbance observer based Ho control for flexible spacecraft with time varying input delay," Advances in Difference Equations, vol. 142, no. 3, pp. 1-12, 2013.

[4] S. Di Gennaro, "Passive attitude control of flexible spacecraft from quaternion measurements," Journal of Optimization Theory and Applications, vol. 116, no. 1, pp. 41-60, 2003.

[5] J. Erdong and S. Zhaowei, "Passivity-based control for a flexible spacecraft in the presence of disturbances," International Journal of Non-Linear Mechanics, vol. 45, no. 4, pp. 348-356, 2010.

[6] H. Bang, C.-K. Ha, and J. H. Kim, "Flexible spacecraft attitude maneuver by application of sliding mode control," Acta Astronautica, vol. 57, no. 11, pp. 841-850, 2005.

[7] Q. L. Hu, Z. Wang, and H. Gao, "Sliding mode and shaped input vibration control of flexible systems," IEEE Transactions on Aerospace and Electronic Systems, vol. 44, no. 2, pp. 503-519, 2008.

[8] Q. Hu, "Robust adaptive sliding mode attitude control and vibration damping of flexible spacecraft subject to unknown disturbance and uncertainty," Transactions of the Institute of Measurement and Control, vol. 34, no. 4, pp. 436-447, 2012.

[9] Z. Chen and J. Huang, "Attitude tracking and disturbance rejection of rigid spacecraft by adaptive control," IEEE Transactions on Automatic Control, vol. 54, no. 3, pp. 600-605, 2009.

[10] B. Li, Q. Hu, and G. Ma, "Extended State Observer based robust attitude control of spacecraft with input saturation," ISA Transactions, vol. 50, pp. 173-182, 2016.

[11] C. Pukdeboon and A. S. I. Zinober, "Control Lyapunov function optimal sliding mode controllers for attitude tracking of spacecraft," Journal of the Franklin Institute, vol. 349, no. 2, pp. 456-475, 2012.

[12] C. Pukdeboon, "Inverse optimal attitude stabilization of flexible spacecraft with actuator saturation," International Journal of Aerospace Engineering, vol. 2016, Article ID 1395952, 14 pages, 2016.

[13] K. Lu, Y. Xia, and M. Fu, "Controller design for rigid spacecraft attitude tracking with actuator saturation," Information Sciences, vol. 220, pp. 343-366, 2013.

[14] H. Du, S. Li, and C. Qian, "Finite-time attitude tracking control of spacecraft with application to attitude synchronization," IEEE Transactions on Automatic Control, vol. 56, no. 11, pp. 2711-2717, 2011.

[15] D. Zhao, S. Li, and F. Gao, "A new terminal sliding mode control for robotic manipulators," International Journal of Control, vol. 82, no. 10, pp. 1804-1813, 2009.

[16] S. Bhat and D. Bernstein, "Finite-time stability of continuous autonomous systems," SIAM Journal on Control and Optimization, vol. 38, no. 3, pp. 751-766, 2000.
[17] E. Moulay and W. Perruquetti, "Finite time stability and stabilization of a class of continuous systems," Journal of Mathematical Analysis and Applications, vol. 323, no. 2, pp. 1430-1443, 2006.

[18] Z. H. Man and X. H. Yu, "Terminal sliding mode control of MIMO linear systems," in Proceedings of the 35th IEEE Transactions on Circuits and Systems I: Fundamental Theory and Applications, vol. 44, pp. 4619-4624, 1996.

[19] Y. Wu, X. Yu, and Z. Man, "Terminal sliding mode control design for uncertain dynamic systems," Systems and Control Letters, vol. 34, no. 2, pp. 281-288, 1998.

[20] S. Wu, G. Radice, Y. Gao, and Z. Sun, "Quaternion-based finite time control for spacecraft attitude tracking," Acta Astronautica, vol. 69, no. 1-2, pp. 48-58, 2011.

[21] Z. Song, H. Li, and K. Sun, "Finite-time control for nonlinear spacecraft attitude based on terminal sliding mode technique," ISA Transactions, vol. 53, no. 1, pp. 117-124, 2014.

[22] A. M. Zou, K. D. Kumar, and Z. G. Hou, "Finite-time attitude tracking control for spacecraft using terminal sliding mode and chebyshev neural network," IEEE Transactions on Systems, Man, and Cybernetics, Part B (Cybernetics), vol. 41, no. 4, pp. 950-963, 2011.

[23] Y. Feng, X. Yu, and Z. Man, "Non-singular terminal sliding mode control of rigid manipulators," Automatica, vol. 38, no. 2, pp. 2159-2167, 2002.

[24] S. Yu, X. Yu, B. Shirinzadeh, and Z. Man, "Continuous finitetime control for roboti manipulators with terminal sliding mode," Automatica, vol. 41, no. 11, pp. 1957-1964, 2005.

[25] C. Pukdeboon and P. Siricharuanun, "Nonsingular terminal sliding mode based finite-time control for spacecraft attitude tracking," International Journal of Control, Automation and Systems, vol. 12, no. 3, pp. 530-540, 2014.

[26] P. M. Tiwari, S. Janardhanan, and M. Nabi, "Rigid spacecraft attitude control using adaptive integral second order sliding mode," in Aerospace Science and Technology, vol. 42, pp. 50-57, 2015.

[27] S. Wu, G. Radice, and Z. Sun, "Robust finite-time control for flexible spacecraft attitude maneuver," Journal of Aerospace Engineering, vol. 27, no. 1, pp. 185-190, 2014.

[28] C. Zhong, Y. Guo, Z. Yu, L. Wang, and Q. Chen, "Finite-time attitude control for flexible spacecraft with unknown bounded disturbance," Institute of Measurement and Control, vol. 5, 2015.

[29] Y. Han, J. D. Biggs, and N. Cui, "Adaptive fault-tolerant control of spacecraft attitude dynamics with actuator failures," Journal of Guidance, Control, and Dynamics, vol. 38, no. 10, pp. 14-22, 2015.

[30] Q. Hu and X. Shao, "Smooth finite-time fault-tolerant attitude tracking control for rigid spacecraft," Aerospace Science and Technology, vol. 55, pp. 144-157, 2016.

[31] K. Lu, Y. Xia, C. Yu, and H. Liu, "Finite-Time Tracking Control of Rigid Spacecraft Under Actuator Saturations and Faults," IEEE Transactions on Automation Science and Engineering, vol. 13, no. 1, pp. 368-391, 2016.

[32] X. Huo, Q. Hu, and B. Xiao, "Finite-time fault tolerant attitude stabilization control for rigid spacecraft," ISA Transactions, vol. 53, no. 2, pp. 241-250, 2014.

[33] B. Xiao and Q. Hu, "Fault-tolerant attitude control for flexible spacecraft without angular velocity magnitude measurement," Journal of Guidance, Control, and Dynamics, vol. 34, no. 5, pp. 1556-1561, 2011. 
[34] R. Zhang, J. Qiao, T. Li, and L. Guo, "Robust fault-tolerant control for flexible spacecraft against partial actuator failures," Nonlinear Dynamics, vol. 76, no. 3, pp. 1753-1760, 2014.

[35] X. Cao, C. Yue, and M. Liu, "Fault-tolerant sliding mode attitude tracking control for flexible spacecraft with disturbance and modeling uncertainty," Nonlinear Dynamics, vol. 76, no. 3, pp. 1753-1760, 2014.

[36] A. Polyakov, "Nonlinear feedback design for fixed-time stabilization of linear control systems," Institute of Electrical and Electronics Engineers Transactions on Automatic Control, vol. 57, no. 8, pp. 2106-2110, 2012.

[37] A. Polyakov, D. Efimov, and W. Perruquetti, "Robust stabilization of MIMO systems in finite/fixed time," International Journal of Robust and Nonlinear Control, vol. 26, no. 1, pp. 6990, 2016.

[38] C. G. Mayhew, R. G. Sanfelice, and A. R. Teel, "On pathlifting mechanisms and unwinding in quaternion-based attitude control," Institute of Electrical and Electronics Engineers Transactions on Automatic Control, vol. 58, no. 5, pp. 1179-1191, 2013.

[39] S. Di Gennaro, "Output stabilization of flexible spacecraft with active vibration suppression," IEEE Transactions on Aerospace and Electronic Systems, vol. 39, no. 3, pp. 747-759, 2003.

[40] G. H. Hardy, J. E. Littlewood, and G. Polya, Inequalities, Cambridge University Press, London, UK, 2nd edition, 1952.

[41] Z. Zuo and L. Tie, "Distributed robust finite-time nonlinear consensus protocols for multi-agent systems," International Journal of Systems Science, vol. 47, no. 6, pp. 1366-1375, 2016.

[42] Z. Zhu, Y. Xia, and M. Fu, "Attitude stabilization of rigid spacecraft with finite-time convergence," International Journal of Robust and Nonlinear Control, vol. 21, no. 6, pp. 686-702, 2011. 


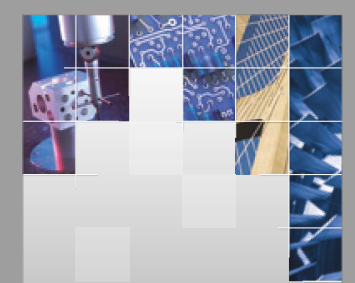

\section{Enfincering}
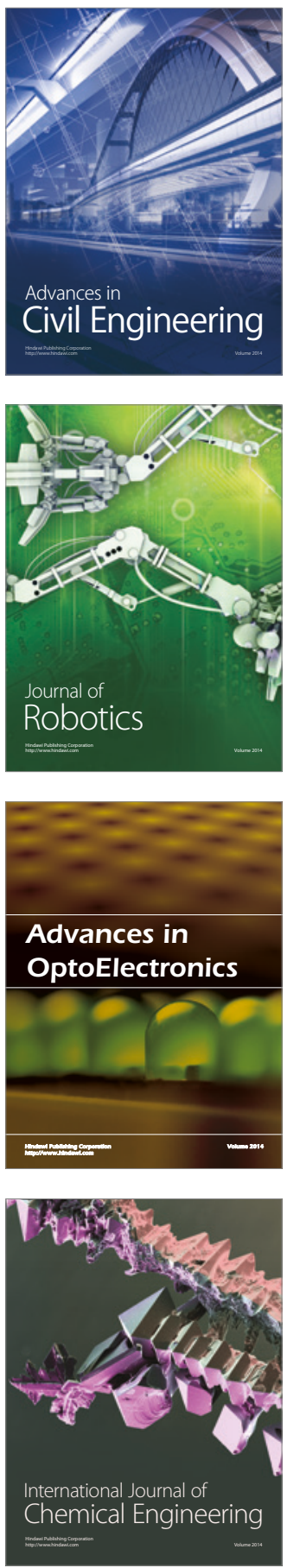

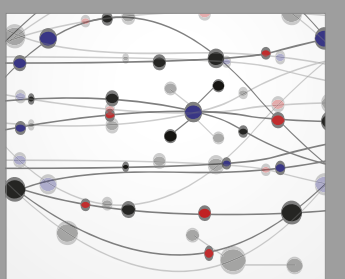

The Scientific World Journal

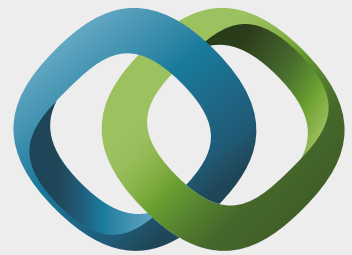

\section{Hindawi}

Submit your manuscripts at

https://www.hindawi.com
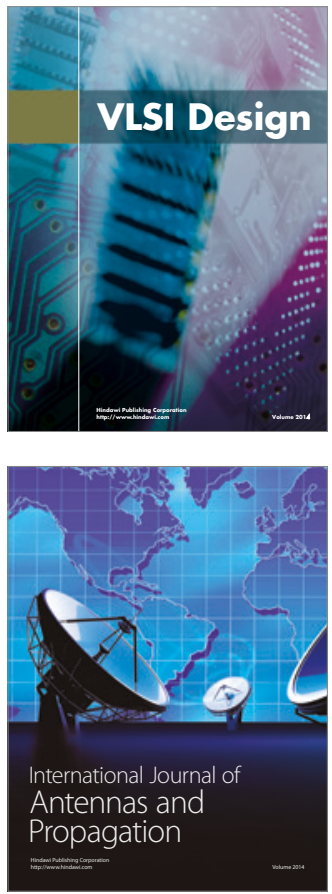

\section{Rotating}

Machinery
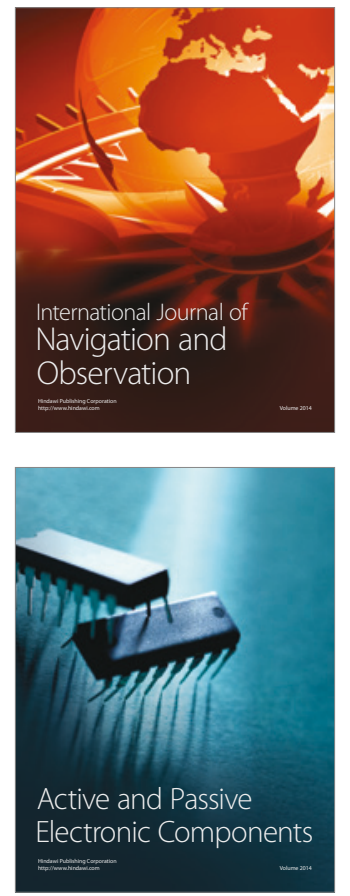
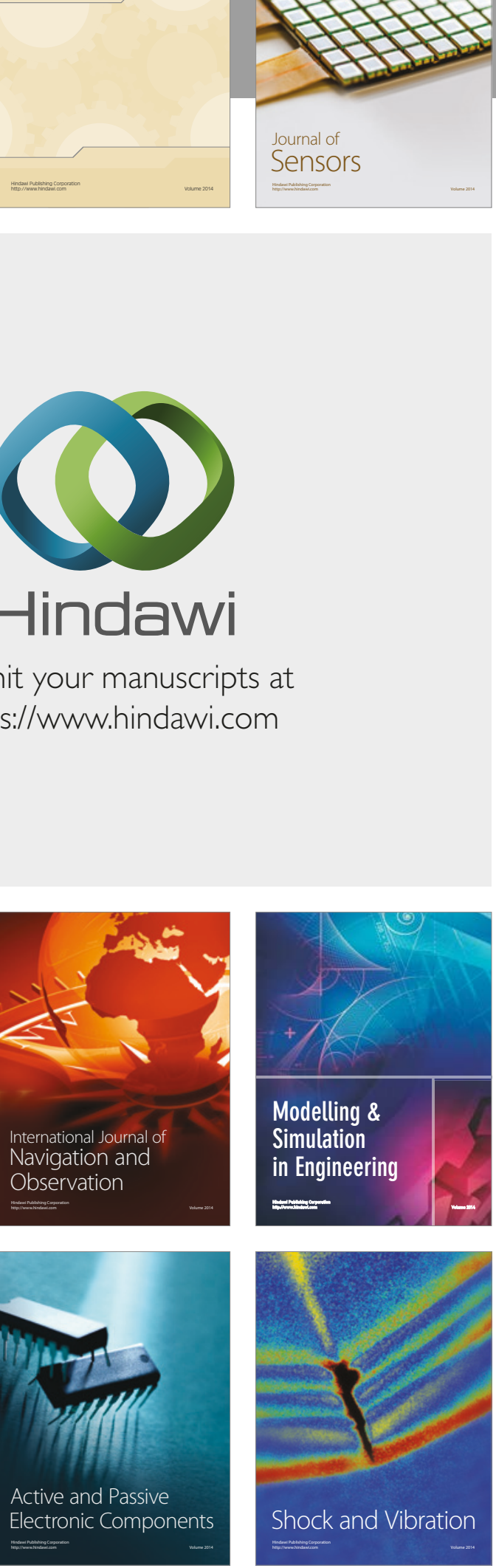
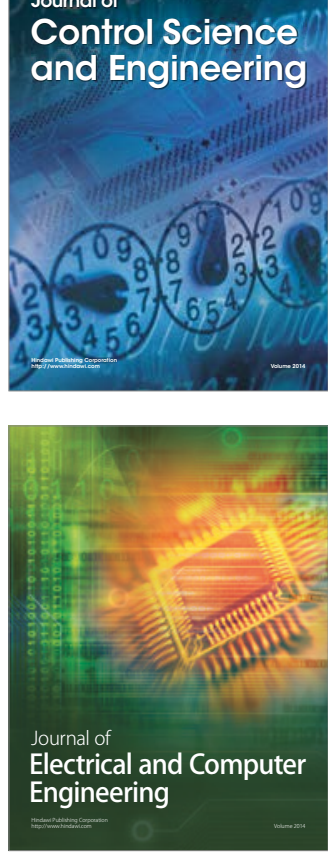

Distributed

Journal of

Control Science

and Engineering
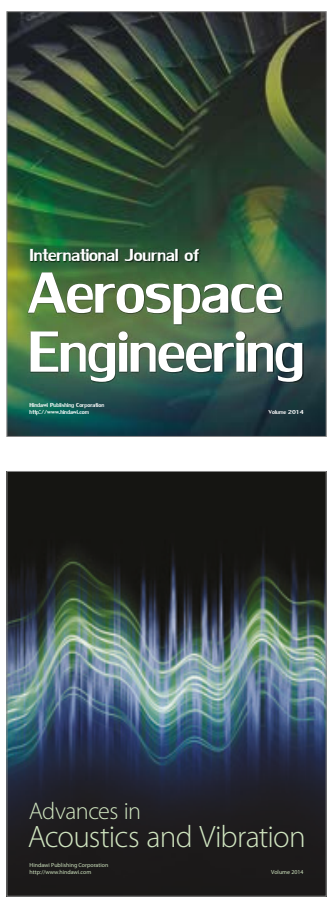

Sensor Networks 